\title{
BANCO DE INSTRUMENTACIÓN PARA EL ACONDICIONAMIENTO Y ADQUISICIÓN DE SEÑALES PROVENIENTES DE UN MOTOR DE COMBUSTIÓN INTERNA
}

\section{INSTRUMENTATION BANK FOR THE CONDITIONING AND ACQUISITION OF SIGNALS FROM AN INTERNAL COMBUSTION ENGINE}

\author{
Juan David Ramírez*, Juan Camilo Mejía Hernández**, Héctor Fabio Quintero***, \\ Edison de Jesús Henao Castañeda****, Carlos Alberto Romero Piedrahita***** y \\ Wilson Pérez Castro******* \\ Universidad Tecnológica de Pereira, Ingeniería Mecánica \\ Carrera 27 \#10-02, Pereira, Risaralda, Colombia. \\ (57) (6) 3137124 \\ E-mail: \{juandaviramireza, j.mejia1, hquinte, edisonhenao, cromero, wilsonmeca $@ @$ utp.edu.co.
}

Resumen: Desde la invención, el Motor de Combustión Interna (MCI) se utiliza en una amplia gama de aplicaciones automovilísticas, aeronáuticas, ferroviarias entre otras. Debido a lo anterior, se han desarrollado diversas técnicas de monitoreo, diagnóstico y mantenimiento para los MCI, las cuales tienen como objetivo mantenerlos en buen estado para reducir costos de reparación, garantizar la disponibilidad y eficiencia. Generalmente, las técnicas de mantenimiento de los MCI se basan en el análisis de señales intrínsecas provenientes de los mismos (temperatura, vibraciones, gases, etc.) y la captura de estas conlleva un enorme trabajo de instrumentación. En este artículo, se presenta detalladamente un robusto y eficiente banco de instrumentación para la captura de señales proveniente de un motor de combustión interna. Las señales capturadas del MCI son temperatura (bloque, aceite, escape y admisión), vibraciones en todos los ejes, presión en la cámara de combustión, posición angular, velocidad y carga. Cada señal fue analizada y acondicionada para ser capturada por el sistema de adquisición de la National Instruments (NI), con el objetivo de cumplir con estándares de comparación establecidos en el estado del arte. Cabe resaltar que, gracias a la metodología implementada todas las señales se capturan de manera simultánea, lo cual permite realizar correlaciones entre las mismas en el tiempo y así encontrar información relevante. Fruto de este trabajo, se presenta un conjunto de señales provenientes de MCI que generalmente no son tomadas en cuenta para realizar diagnósticos y muchos menos analizadas de manera conjunta. Además, se exhibe una base de datos con todas las señales cambiando las condiciones de trabajo (velocidad y carga) del MCI, la cual puede ser utilizada para casos de estudio.

Palabras clave: Motor de Combustión Interna, Técnicas de monitoreo, Instrumentación, National Instruments, Condiciones de trabajo.

\begin{abstract}
Abstract: Since the invention, the Internal Combustion Engine (ICM) has been used in a wide range of automotive, aeronautical and railway applications, among others. Due to the above, several monitoring, diagnosis and maintenance techniques have been developed for the MCI, which aim to keep them in good condition to reduce repair costs, guarantee availability and efficiency. Generally, the MCI maintenance techniques are based on the analysis of intrinsic signals coming from them (temperature, vibrations, gases, etc.) and the capture of these involves an enormous work of instrumentation. In this article, a robust and efficient instrumentation bank for the capture of signals coming from an internal combustion engine is presented in detail. The signals captured from the MCI are temperature (block, oil, exhaust and intake), vibrations on all axes, pressure (combustion chamber and intake air), angular position, speed and load. Each signal was
\end{abstract}


analyzed and conditioned to be captured by the acquisition system of the National Instruments (NI), in order to comply with standards of comparison established in the state of the art. It should be noted that, thanks to the implemented methodology, all the signals are captured simultaneously, which allows to make correlations between them over time and thus find relevant information. As a result of this work, we present a set of signals from MCI that are not generally taken into account to make diagnoses and much less analyzed jointly. In addition, a database with all the signals is displayed, changing the working conditions (speed and load) of the MCI, which can be used for case studies.

Keywords: Internal combustion engine, monitoring techniques, instrumentation, National Instruments, working conditions.

\section{INTRODUCCIÓN}

En la mayor parte de las aplicaciones, los Motores de Combustión Interna (MCI) tienen que hacer frente a condiciones operativas variables. Debido a esto, se han desarrollado diferentes ambientes controlados para realizar pruebas en los MCI, en las cuales se puede registrar y almacenar información relevante que permite identificar las mejores condiciones de operación (Jindal, 2010). Dichas condiciones, son una aproximación de las verdaderas configuraciones que deben tener un MCI para funcionar de manera óptima en ambientes no controlados. Un MCI optimizado adquiere ventajas como reducción de combustible, máximo aprovechamiento de la potencia y una reducción de costos de reparación (Muralidharan, 2010, Jairo, 2018). Este enfoque se conoce como mantenimiento preventivo y se realiza en la actualidad mediante una serie de rutinas de inspección realizadas a unos intervalos de tiempo determinados.

Diferentes estudios se han enfocado en la búsqueda de las condiciones óptimas de los MCI. Chaican et al., (2010) buscan relaciones aire-combustión óptima para diferentes combustibles en un motor cuatro tiempos. Además, Rodríguez et al., (2018) y Yousufuddina et al., (2015) realizaron búsquedas de variaciones de combustibles para encontrar las mayores compresiones en cámara. Camarillo et al., (2008) y Kumar et al., (2011) comparan diferentes combinaciones de combustible para buscar los mejores parámetros de par y potencia efectiva. Sin embargo, todos los trabajos mencionados usan una pequeña cantidad de señales, lo cual omite correlaciones de fenómenos físicos del motor que pueden ayudar a encontrar de manera más eficiente los parámetros óptimos para el MCI. En este trabajo se presenta un robusto banco de instrumentación para el acondicionamiento y adquisición de múltiples señales provenientes de un motor MCI. Los sensores, circuitos de acondicionamiento, sistemas de adquisición y protocolos de captura fueron escogidos específicamente para lograr una correcta recolección de la información que puede ofrecer los fenómenos físicos que posee el motor.

El resto del articulo está organizado de la siguiente manera: En la sección 2 se describe el banco de instrumentación para la captura de señales provenientes de un MCI. Luego, en la sección 3 se especifica todos los protocolos que se usaron para la creación de una base de datos fruto de esta investigación. Por último, las conclusiones se describen en la sección 4.

\section{BANCO DE INSTRUMENTACIÓN}

En esta sección se detalla un Banco de Instrumentación (BI) para el acondicionamiento, adquisición y almacenamiento de diferentes señales provenientes de un MCI. Todo el sistema esta detallado en la Fig. 1.

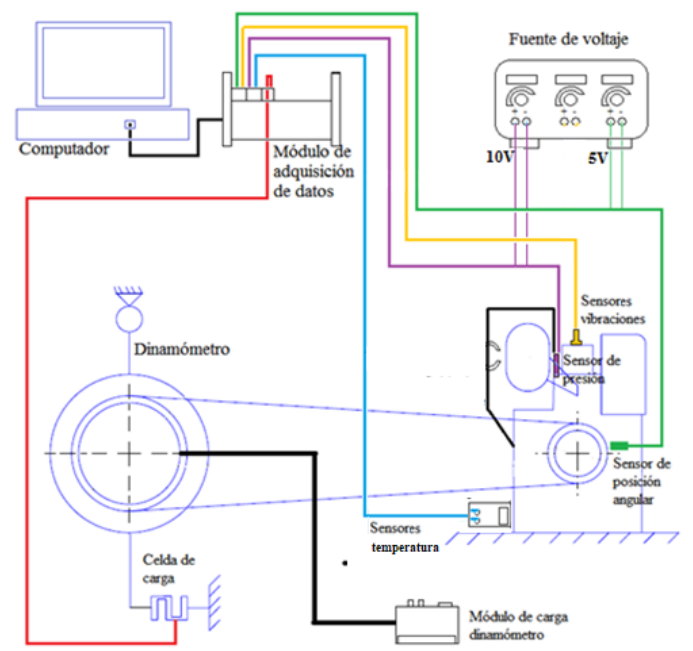

Fig. 1. Banco de instrumentación para la captura de señales provenientes de un MCI.

El BI de la Fig. 1 se compone de una seria de sensores y acondicionamientos para la captura de temperatura (bloque, aceite, escape y admisión), 
vibraciones en todos los ejes, presión en cámara de combustión, posición angular, velocidad y carga. Dichas señales son adquiridas por una serie de tarjetas de alta precisión de la National Instruments (NI), las cuales fueron escogidas basándose en las características intrínsecas de los sensores y el fenómeno físico. Luego, se sincronizan todas las tarjetas mediante un chasis de la NI y se crean los protocolos de adquisición usando la herramienta de desarrollo Matlab.

\subsection{Temperatura}

La temperatura de un MCI es bastante elevada en diferentes partes del mismo, por lo cual se requirió un tipo de sensor robusto y resistente. Para esta aplicación se usó diferentes termocuplas ubicadas en puntos estratégicos del motor. La primera de tipo $\mathrm{K}$ está en la admisión, la cual sirve para identificar la energía calórica que puede ofrecer el combustible. La segunda tipo K, está ubicada en bloque y permite monitorear la temperatura del MCI al generar el movimiento. La tercera tipo J, posicionada de manera en el aceite para vigilar variaciones importantes de lubricación. La última de tipo $\mathrm{K}$, ubicada en el escape y mide la temperatura de los gases que bota el MCI. Un diagrama de las ubicaciones de las termocuplas se aprecia en la Fig. 2.

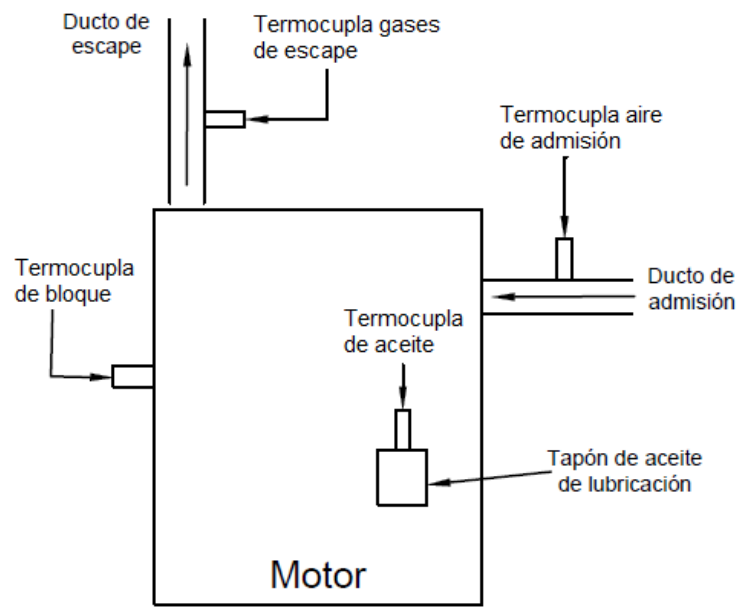

Fig. 2. Termocuplas ubicadas en el MCI

\subsection{Vibración}

Con el fin de adquirir señales de vibraciones en el motor, se ubicaron tres placas planas de acero en los diferentes ejes, con el objetivo de proporcionar información de todos los desplazamientos que sufre el MCI. Los sensores usados son de la marca CTC modelo AC102-1A con sensibilidad 100mV/g y sus ubicaciones se pueden apreciar en la Fig. 3.

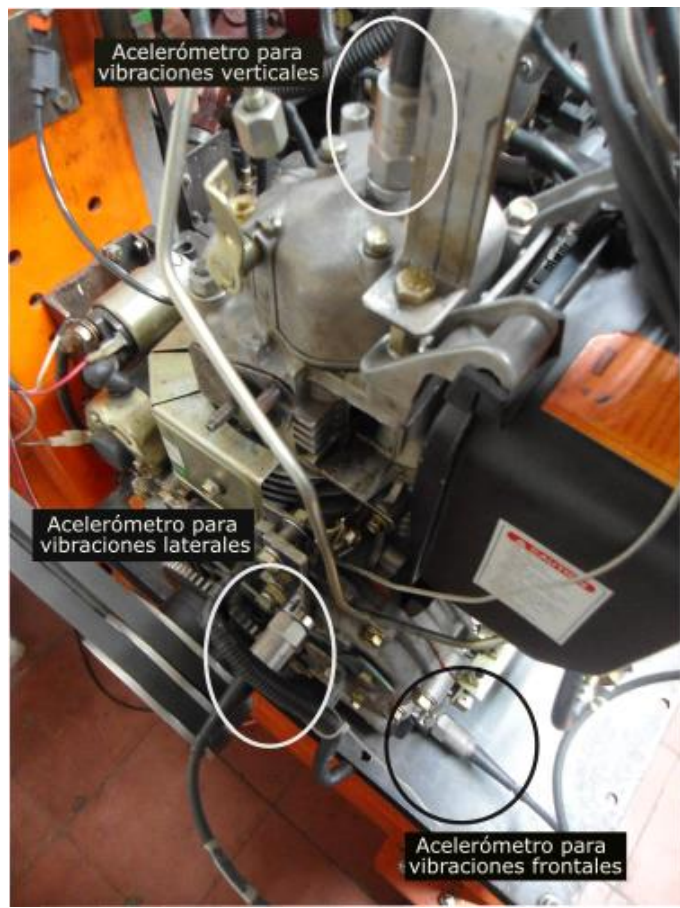

Fig. 3. Ubicación de los sensores de vibración

Las vibraciones mecánicas se usan en una amplia gama de diagnósticos debido a que poseen mucha información relevante del sistema. Por lo tanto, cual se implementaron en todos los ejes del MCI. En el eje $X$ se capturan vibraciones frontales, en el eje $\mathrm{Y}$ vibraciones verticales $\mathrm{y}$ en el eje $\mathrm{Z}$ vibraciones laterales. $\mathrm{El}$ sensor que más información relevante contiene es el ubicado en el eje $\mathrm{Y}$, puesto que va en el mismo sentido que el movimiento del pistón.

\subsection{Presión}

En un MCI que trabaja en óptimas condiciones, la mezcla de combustible y aire se debería quemar de forma uniforme y progresiva, puesto que una detonación violenta de la mezcla provoca un estado de marcha desigual. Dicho fenómeno puede monitorearse en la cámara de combustión, por lo cual se implementó un sensor para la medición de la presión. La implementación comenzó con una modificación de la culata del motor y luego el posicionamiento de un sensor Optrand D822J6-SP, el cual se apreciar en la Fig.4. 

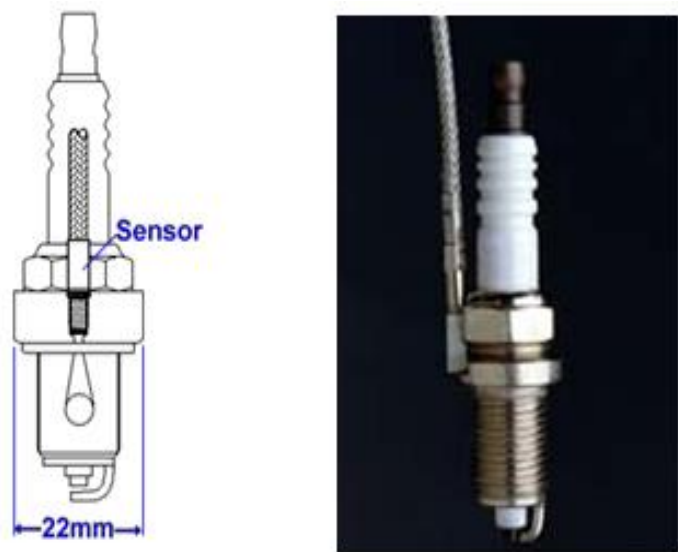

Fig. 4. Sensor de presión Optrand D822J6-SP utilizado en cámara de combustión.

El sensor tiene forma de bujía y permite observar la variación se presión que permite identificar comportamientos anormales como combustiónes espontáneas de la mezcla que queda en la cámara. Esto repercute en el sistema de refrigeración, ya que este no puede eliminar todo el calor producido, lo que causa calentamientos excesivos del MCI.

\subsection{Velocidad y posición angular}

En la polea ubicada en el eje de salida del motor, se instaló una rueda dentada (encoder) para medir la posición angular de cigüeñal y se adhirió una cinta reflectiva para medir las RPM con un tacómetro digital. La rueda dentada instalada con este propósito es fónica 60-2, lo cual indica que, a la rueda original de 60 dientes, se le retiran dos (2) con el fin de ubicar cada revolución gráficamente. Luego, se instaló un sensor de efecto Hall, el cual mide campo magnético o corriente para determinar la posición angular del cigueñal mediante pulsos creados por un voltaje proporcional al producto de la fuerza del campo magnético y de la corriente. El posicionamiento del sensor se puede ver en la Fig. 5.

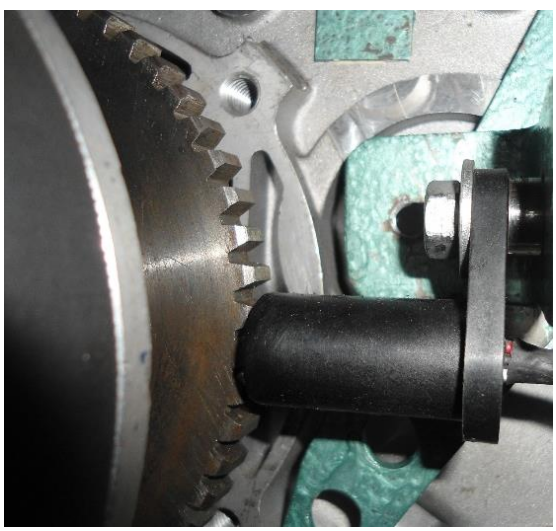

Fig. 5. Sensor de posición efecto Hall instalado en el motor del banco de pruebas
El sensor permite apreciar una señal cuadrada, la cual cambia de valor cada que un diente se acerca. Dicha señal y la frecuencia de muestreo de la tarjeta NI permite calcular en un espacio de tiempo la revoluciones por minuto del MCI.

\subsection{Carga}

La carga del motor se modifica usando un dinamómetro de "Corrientes de Eddy", el cual se puede apreciar en la figura 6. Su principio de funcionamiento es un mecanismo de freno que utiliza la inducción electromagnética para crear el fenómeno conocido como corriente de Foucault, creando una serie de resistencias, aprovechadas para frenar el motor. Su conexión con el motor se visualiza en la Fig. 7.

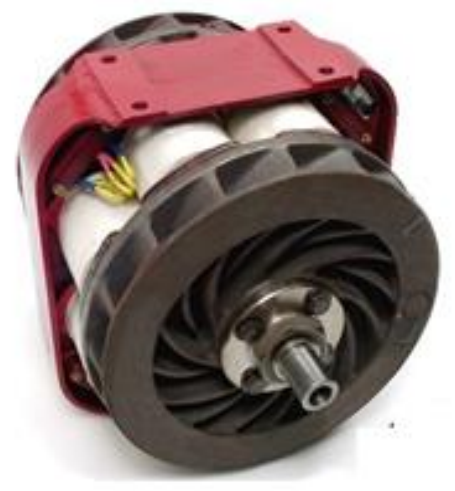

Fig. 6. Dinamómetro CFK-200.

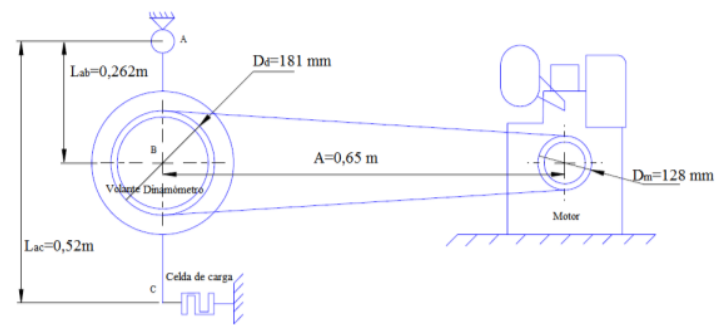

Fig. 7. Diagrama de cuerpo libre del motor y el dinamómetro.

Gracias a este dinamómetro se puede calcular y variar la carga a la que está sometido el MCI. Lo anterior se logra midiendo la deformación en una celda compuesta por 4 galgas extensiométricas y acondicionadas con un puente de Wheatstone. La señal resultante es la fuerza de oposición del dinamómetro al motor y se expresa en gramos.

\subsection{Sistema de adquisición National Instruments}

Para la adquisición de las señales provenientes del MCI se utilizaron diferentes tarjetas de la NI 
escogidas basándose en las características de los sensores y el fenómeno físico. El sistema de adquisición para cada señal se puede apreciar en la Fig. 8.

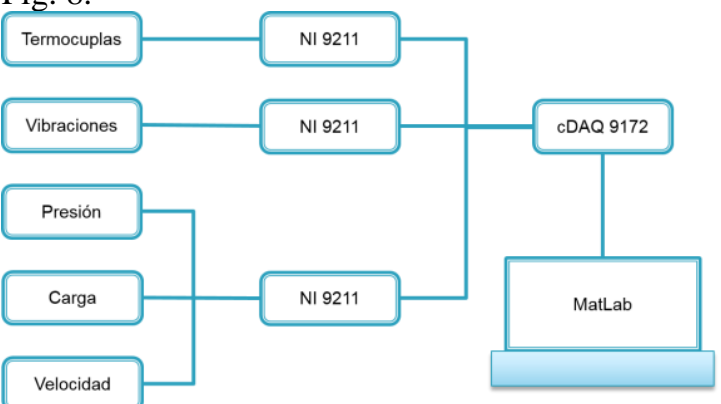

Fig. 8. Sistema de adquisición del BI

Para las termocuplas se usó la tarjeta NI 9211 (4 muestras por segundo). Las vibraciones usaron la tarjeta NI 9234 (51200 muestras por segundo por canal). La presión, la carga y la posición angular se adquirieron con la tarjeta NI 9222 (51200 muestras por segundo por canal). Luego, las tres tarjetas se ubicaron en el chasis cDAQ-9172 y se estableció el protocolo y configuraciones en la herramienta de desarrollo MatLab.

\section{BASE DE DATOS}

La base de datos se obtuvo adquiriendo señales provenientes de un motor CHANGFA $186 \mathrm{~F}$, el cual funciona con Diesel $y$ es normalmente utilizado en obras civiles, generación de energía eléctrica y en labores agrícolas. Dicho motor se puede apreciar en la Fig. 9.

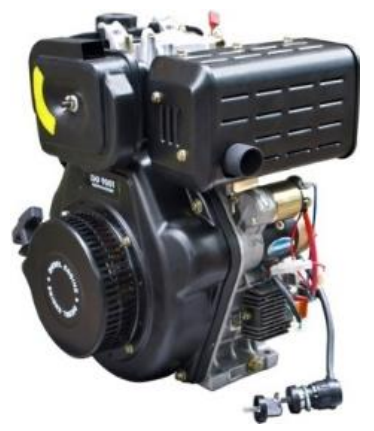

Fig. 9. Motor CHANGFA 186 F

El MCI mencionado posee la ventaja de estar modificado para variar de manera controlada diferentes factores que tienen como propósito crear una base de datos robusta. Los factores de estudio elegidos fueron:

- Velocidad en RPM:

$$
\begin{array}{cc}
\text { ○ } & 1200 \\
\circ & 1800 \\
\circ & 2400
\end{array}
$$

$$
\begin{array}{ll}
\text { ○ } & 3000 \\
\text { ○ } & 3600
\end{array}
$$

- Nivel de carga (Amperios)

$$
\begin{array}{ll}
\circ & \text { Baja } \\
\circ & \text { Media } \\
\circ & \text { Alta }
\end{array}
$$

Se utilizarán un conjunto de 15 experimentos variando los factores de estudio, con el objetivo de evaluar el desempeño del motor usando un solo tipo de combustible. La matriz de experimento utilizada se muestra en la Tabla 1.

Tabla 1. Matriz de experimento

\begin{tabular}{ccc}
\hline & Experimento & \\
\hline Carga & RPM & Tratamiento \\
\hline & 1200 & 1 \\
Baja (0,5 A) & 1800 & 2 \\
& 3400 & 3 \\
& 3600 & 4 \\
& 1200 & 5 \\
\hline \multirow{3}{*}{ Media (1 A) } & 1800 & 6 \\
& 3400 & 7 \\
& 3000 & 8 \\
& 3600 & 9 \\
& 1200 & 11 \\
& 1800 & 12 \\
& 2400 & 13 \\
& 3000 & 14 \\
& 3600 & 15 \\
\hline
\end{tabular}

La base de datos se adquirió manejando una homogeneidad en cuanto a frecuencia de muestreo y duración. Para las temperaturas 4 muestras por segundo y para las demás señales 51200 muestras por segundo.

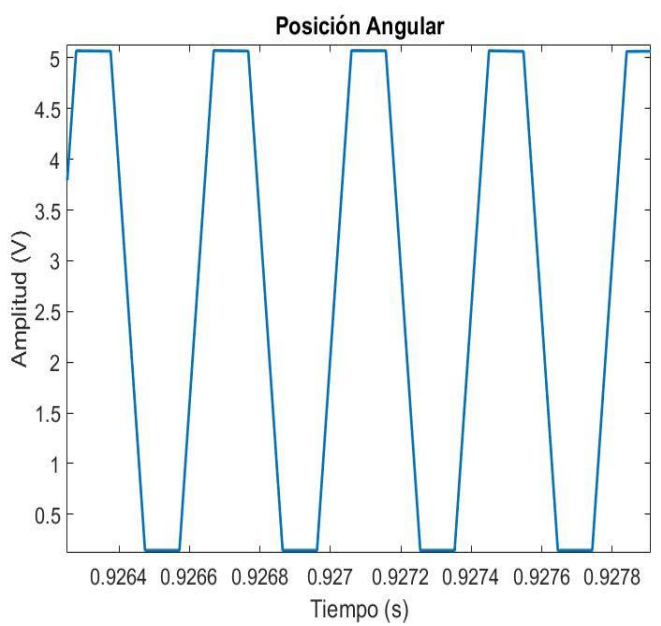


Fig. 13. Señal posición angular
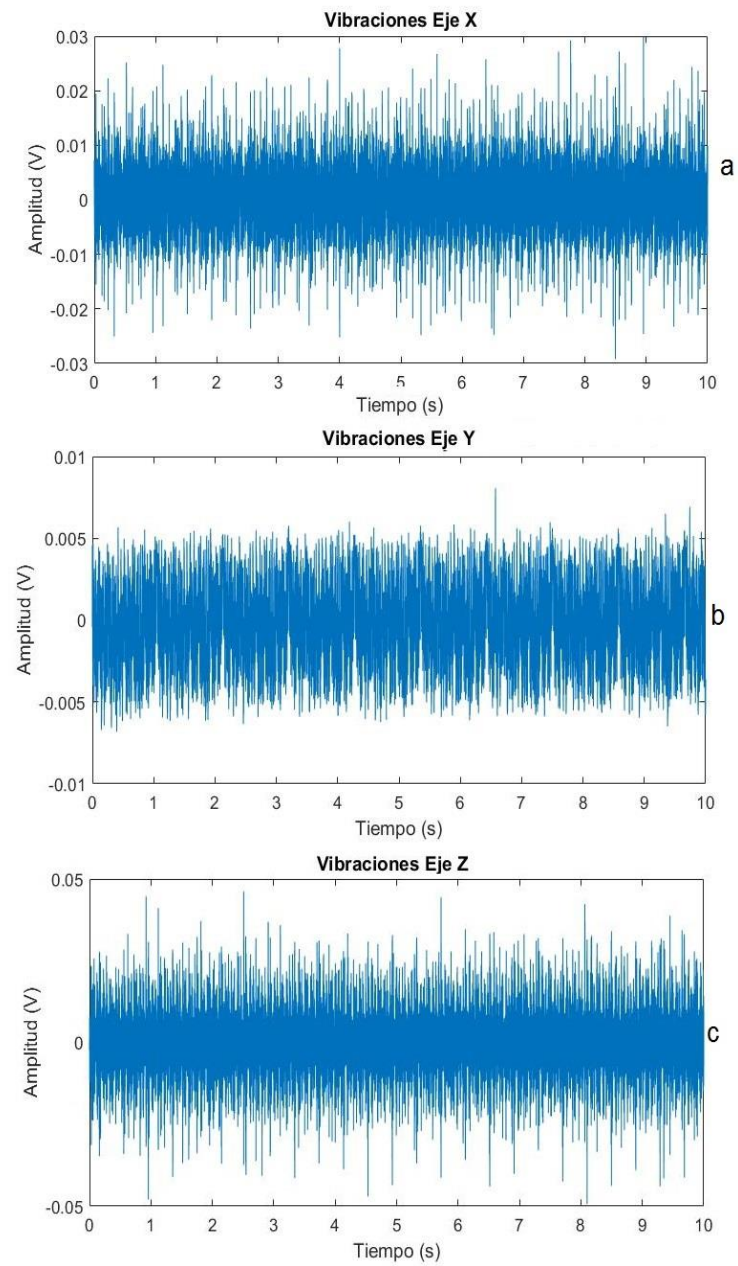

Fig. 11. Señales de vibración. a) Eje X. b) Eje $Y$. c) Eje Z.

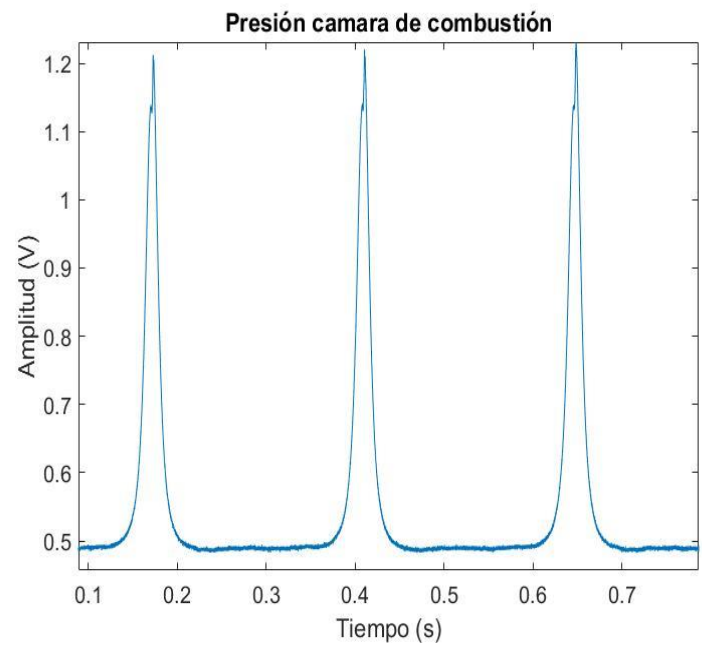

Fig. 12. Señal de presión
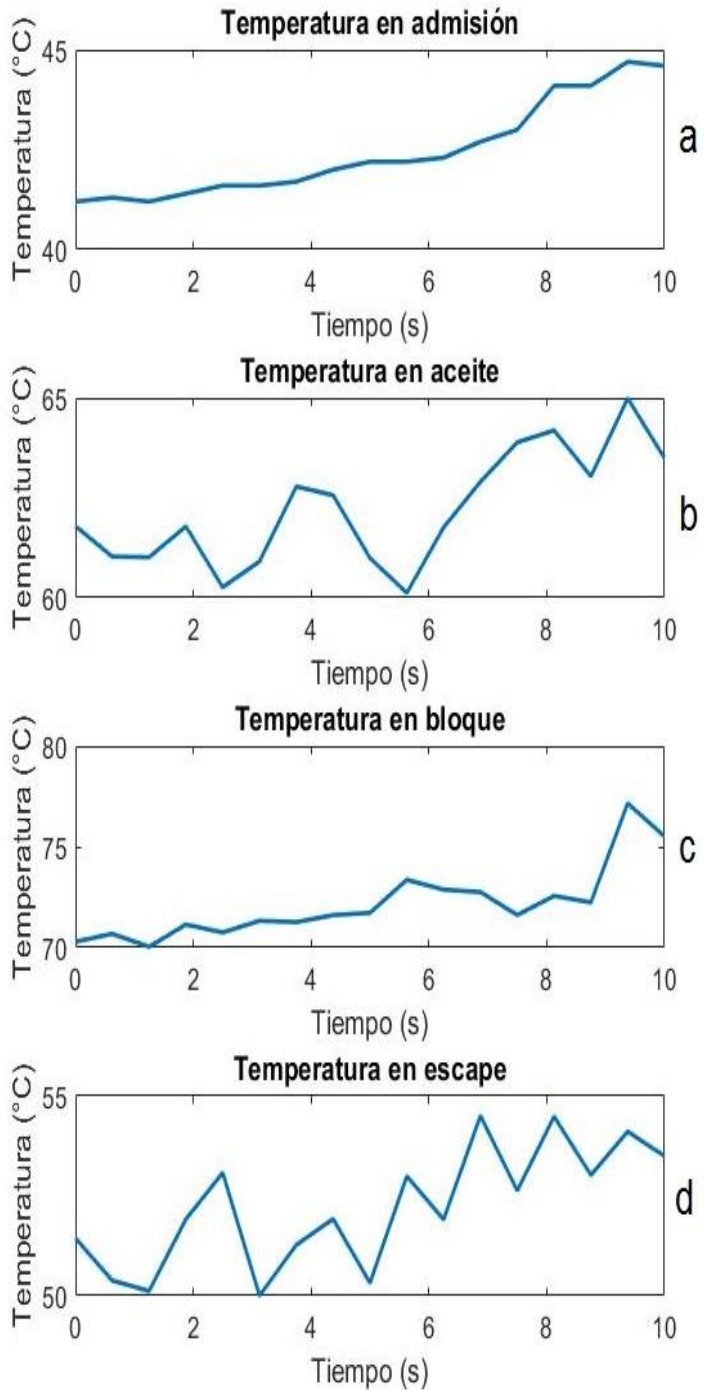

Fig. 10. Señales de temperatura. a) Admisión. b) Aceite. c) Bloque. d) Escape.

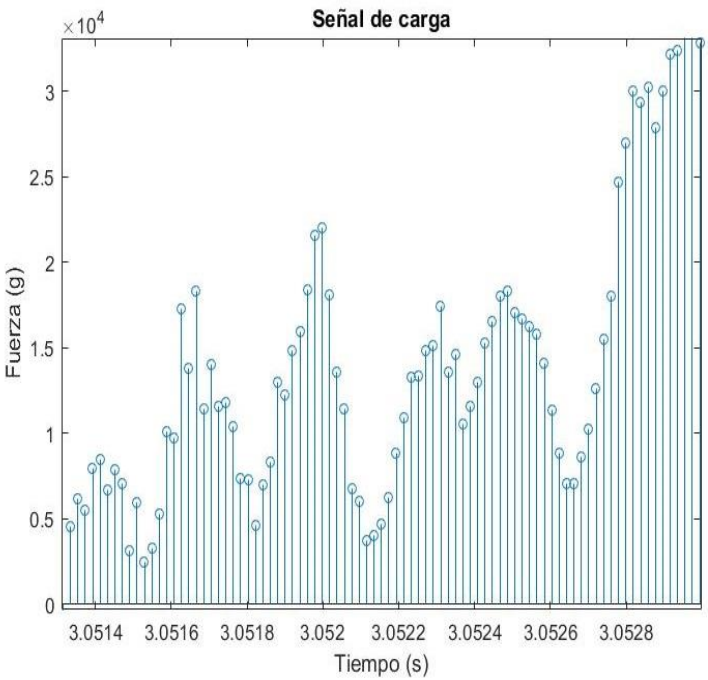

Fig. 14. Señal de carga 
Las señales fueron adquiridas de manera sincronizada y se logra capturar el fenómeno físico garantizando la recolección de la información que contiene acerca del MCI.

\section{CONCLUSIONS}

En este artículo se presenta un robusto BI para el acondicionamiento y adquisición de múltiples señales provenientes de un motor MCI. Las señales capturadas son temperatura (bloque, aceite, escape y admisión), vibraciones en todos los ejes, presión en la cámara de combustión, posición angular, velocidad y carga. Las señales son capturadas con sistemas de adquisición de la NI, con el objetivo de tener garantías de acondicionamiento y frecuencia de muestreo. El BI logra capturar todos los fenómenos físicos de manera sincronizadas y se pueden relacionar entre sí para encontrar las mejores condiciones de trabajo del MCI. Gracias a este BI se creó una base de datos variando las condiciones de trabajo del MCI para ser utilizada como caso de estudio. Un primer trabajo futuro de esta investigación es la instrumentación de otras señales del MCI como presión del múltiple de entrada y salida, emisiones de gases, punto muerto superior y opacidad. Por último, se espera implementar diferentes metodologías de análisis de señales para encontrar patrones dinámicos que infieren las condiciones óptimas de trabajo.

\section{RECONOCIMIENTO}

Los autores agradecen al Master en Ingeniería Eléctrica y Mecánica de la Universidad Tecnológica de Pereira por su apoyo a lo largo de la investigación. Además, queremos agradecer a COLCIENCIAS por apoyar el proyecto titulado: "Desarrollo de un sistema de monitoreo para el análisis energético y de condición de emisiones en motores de combustión interna diésel con base en técnicas no intrusivas" con el código 1110-77657801, a través del cual se desarrolló la investigación descrita en este artículo.

\section{REFERENCES}

S. Jindal, B.P. Nandwana, N.S. Rathore y V. Vashistha. Experimental investigation of the effect of compression ratio and injection pressure in a direct injection diesel engine running on Jatropha methyl ester. Applied Thermal Engineering. Vol 30. 2010. 442-448.
K. Muralidharan, D. Vasudevan. Performance, emission and combustion characteristics of a variable compression ratio engine using methyl esters of waste cooking oil and diesel blends. Applied Energy. Vol. 88. 2010. 3959-3968.

Miqdam T. Chaichan y Adel M. Saleh. Practical investigation of single cylinder spark ignition engine performance operated with various hydrocarbon fuels and hydrogen. Journal of Engineering and Development. Vol. 14, No 2. 2010. 183-197.

Juan Felipe Rodríguez Rueda. Caracterización, puesta a punto y análisis de emisiones generadas en un motor de combustión interna HCCI operado con etanol anhidro (E100) y etanol hidratado (E80). Grupo de investigación en combustibles alternativos, energía y protección del medio ambiente GICAEPMA, Universidad Nacional de Colombia. 2015.

Milen Balbis Morejón, Francisco García Reina, Juan Jose. Caracterización energética del funcionamiento de un equipo de aire acondicionado en un local dado. Revista Colombiana de Tecnologías de Avanzada. Vol. 2. 43-60. 2018

D.C. Rakopoulos, C.D. Rakopoulos, E.G. Giakoumis, R.G. Papagiannakis, D.C. Kyritsis. Experimental-stochastic investigation of the combustion cyclicvariability in HSDI diesel engine using ethanol-diesel fuel blends. ELSEVIER. Fuel. Vol 87.2008. 1478-1491.

M. Pandian, S.P. Sivapirakasam, M. Udayakumar. Investigation on the effect of injection system parameters on performance and emission characteristics of a twin cylinder compression ignition direct injection engine fuelled with pongamia biodiesel-diesel blend using response surface methodology. ELSEVIER. Applied Energy. Vol 88. 2011. 2663-2676.

Jairo Gómez Tapia, Camilo Leonardo. Análisis de prospectiva del sector energético de Colombia, para la integración de fuentes fotovoltaicas en los sistemas de distribución de energía eléctrica aplicando una revisión en bases de datos científicas. Revista Colombiana de Tecnologías de Avanzada. Vol. 2. 32-43. 2018 